\title{
Un dispositif universitaire centré sur un travail réflexif d'exploration du vécu subjectif : formation et transformation de soi
}

A university scheme focused on reflective work exploring subjective experience:

education and self-transformation

\section{Nadine Faingold}

\section{(2) OpenEdition}

Journals

Édition électronique

URL : http://journals.openedition.org/rechercheformation/2499

DOI : 10.4000/rechercheformation.2499

ISSN : 1968-3936

Éditeur

ENS Éditions

\section{Édition imprimée}

Date de publication : 31 décembre 2015

Pagination : 47-62

ISBN : 978-2-84788-867-6

ISSN : 0988-1824

\section{Référence électronique}

Nadine Faingold, «Un dispositif universitaire centré sur un travail réflexif d'exploration du vécu subjectif : formation et transformation de soi », Recherche et formation [En ligne], 80 | 2015, mis en ligne le 31 décembre 2018, consulté le 05 janvier 2021. URL : http://journals.openedition.org/ rechercheformation/2499; DOI : https://doi.org/10.4000/rechercheformation.2499 


\title{
Un dispositif universitaire centré sur un travail réflexif d'exploration du vécu subjectif : formation et transformation de soi
}

\author{
$>$ Nadine Faingold \\ CNAM, Centre de recherche sur la formation
}

RÉSUMÉ : Le master "Formation de formateurs à l'accompagnement professionnel" de l'université de Cergy-Pontoise s'adresse à des professionnels des métiers de la relation, et privilégie une approche en première personne, expérientielle et réflexive. Dans ce cadre a été proposé un dispositif centré sur l'explicitation puis le décryptage des enjeux et des valeurs impliqués dans les pratiques professionnelles. Les étudiants sont invités à mettre en place un accompagnement professionnel mobilisant des temps d'entretiens d'explicitation et de décryptage, puis à opérer un retour réflexif sur leur posture et leur vécu subjectif au cours de cet accompagnement.

MOTS-CLÉS : formateur, expérience professionnelle, relations interpersonnelles, éducation des adultes, méthodologie

\section{Introduction}

Le Master 2 (M2) "Formation de formateurs à l'accompagnement professionnel" de l'université de Cergy-Pontoise a été conçu par Martine Agogué, Nicole Clerc et moi-même à destination des professionnels de la relation. Il vise le développement de compétences transversales spécifiques au métier de formateur d'adultes. Nos étudiants sont donc des enseignants, des infirmiers, des travailleurs sociaux qui souhaitent devenir formateurs. Mais la majorité d'entre eux sont déjà tuteurs de jeunes collègues débutant dans le métier, et même souvent déjà formateurs en instituts de formation. Ils s'inscrivent à partir d'un besoin en formation portant sur la fonction d'accompagnement et l'analyse des pratiques en entretien duel ou en groupe.

Ce cursus de M2 se déroule sur deux années et a pour spécificité de privilégier une formation expérientielle et réflexive. Les groupes sont de vingt étudiants au maximum, afin de permettre un suivi personnalisé des parcours de formation. La maquette comprend des unités d'enseignement sur la conduite d'entretien, l'animation de groupes d'analyse de pratique, l'aide au changement, l'analyse institutionnelle, 
l'ingénierie de formation, la communication et l'interaction dans les groupes, ainsi que des séminaires sur les méthodologies qualitatives et des séminaires de recherche. L'ensemble des évaluations privilégient un retour réflexif sur la pratique et une écriture en "je". Le mémoire de fin de master prend nécessairement appui sur la mise en place par l'étudiant d'un accompagnement professionnel individuel ou collectif au cours de la seconde année. C'est dans ce cadre qu'a pu être proposé un dispositif spécifique, visant à l'appropriation parallèle de compétences introspectives et de compétences d'accompagnement. L'objet de cet article est de décrire ce dispositif de formation qui a été conçu à partir de mes travaux de recherche' sur l'analyse de l'activité et la construction de l'identité professionnelle dans les métiers de l'humain. Le parcours en deux ans comporte 4 Unités d'enseignement (UE) de 21 heures (soit 7 séances de 3 heures par UE) : conduite d'entretien, ateliers de professionnalisation, exploration des parties de soi et aide au changement, identité professionnelle et co-identités. La progression est entièrement orientée vers l'exploration du vécu subjectif et comporte d'abord une formation à l'entretien d'explicitation, puis une formation à l'entretien de décryptage et propose enfin un travail de recherche introspective sur les enjeux identitaires en situation professionnelle. Pour chaque UE, un travail réflexif est demandé pour évaluation, à partir d'entretiens transcrits et analysés. Le début de cet article présente la logique temporelle de cette progression, puis la structure expérientielle des mises en situation. Ensuite, deux exemples, choisis parmi une quarantaine de travaux d'étudiants comme étant particulièrement significatifs, visent à illustrer l'exploration du vécu subjectif dans un but d'aide à la prise de conscience et de formation à une posture réflexive.

\section{Quelle logique de progression?}

La question de départ était la suivante : comment former nos étudiants à la conduite d'entretiens maïeutiques (Galvani, 2014) conçus comme une aide à la prise de conscience par le sujet lui-même de ses ressources, mais aussi des points à travailler en situation professionnelle? Une formation à l'explicitation de l'action et au décryptage du sens me paraissait répondre à cette triple exigence : mettre en place une démarche introspective, accompagner le déroulement de l'action mais aussi l'accueil de l'émotion, dans une aide à la description du vécu subjectif de moments professionnels dans leur complexité. À ceci s'ajoutait l'intérêt d'une formation expérientielle donnant lieu à différents retours sur soi par la prise en compte des observations, l'écoute des enregistrements, la transcription des entretiens, et l'écriture réflexive à des fins d'évaluation des compétences acquises.

1 Voir les rapports de recherche suivants : Construction de l'identité professionnelle des conseillers pédagogiques du premier degré (1998-1999) et Construction de l'identité professionnelle des enseignants spécialisés chargés de l'aide pédagogique auprès des enfants en difficulté (2000-2003) édités par l'IUFM de l'académie de Versailles, coord. N. Faingold; Analyse de l'activité des éducateurs de la PJJ (2009) et Dire le travail éducatif édités par ENPJJ/CNAM, coord. N. Faingold, avec les contributions de S. Debris, P.A. Dupuis, A. Flye Sainte-Marie, R. Wittorski. 
Par ailleurs, le choix d'une formation par mises en situation d'entretien en groupes de trois comportant un accompagnant, un accompagné, et un observateur, avec une rotation systématique de ces trois rôles au fil des séances, répondait à trois hypothèses de départ. La première hypothèse était la nécessité pour savoir accompagner autrui d'expérimenter soi-même des situations en tant qu'accompagné, et pour savoir accueillir les émotions de l'autre d'avoir appris à accueillir les siennes. La seconde hypothèse était qu'il importait de commencer par installer chacun sur la reconnaissance de ses ressources propres en tant que point d'appui pour un développement professionnel, avant d'aborder la clarification des éléments de problématique personnelle susceptibles d'interférer dans la pratique du métier. La troisième hypothèse était qu'il fallait créer un climat de confiance suffisant dans le groupe pour rendre possible une parole authentique de chacun sur ses vulnérabilités et l'acceptation d'être accompagné en sécurité dans cette exploration introspective.

Le point de départ de ce dispositif a donc été une formation de base à l'entretien d'explicitation, parce que le rôle d'intervieweur dans l'approche de Pierre Vermersch induit nécessairement un travail sur soi et un changement assez radical dans la posture d'écoute et d'accompagnement (Faingold, 2012). Mais le fait que la description recherchée soit spécifiquement orientée vers des vécus professionnels dans les métiers de la relation imposait de dépasser une approche principalement cognitive pour tenir compte de la coloration émotionnelle des situations évoquées.

Traditionnellement, la demande des professionnels des métiers de l'éducation, du travail social ou du soin en matière d'analyse de pratiques professionnelles est prioritairement orientée vers ce qui leur pose problème, et donc vers des situations difficiles qu'il convient de clarifier. D’où l'impérieuse nécessité de prévoir dès la première année une formation à la conduite de groupes d'analyse de pratique, incluant nécessairement pour nos étudiants des mises en situation d'analyse collective de leurs propres pratiques professionnelles. Ces enseignements sont assurés par mes collègues psychosociologues dès le début de la première année et tout au long du Master. En complémentarité, il s'agissait de construire un dispositif permettant de former nos étudiants à la conduite d'entretien en relation duelle.

Mon choix a donc été de commencer en première année par l'exploration de situations positives, des situations ressource, permettant la prise de conscience des compétences et des valeurs incarnées dans ces moments de pratique, et de reporter à la seconde année du master le travail en entretien sur des vécus problématiques. En effet, l'entretien d'explicitation, en ce qu'il oriente le sujet vers un revécu de ce qui s'est joué pour lui dans un moment passé, remet en contact avec l'activité dans toute sa complexité, à savoir les aspects cognitifs de son fonctionnement, l'enchaînement des prises d'information et des prises de décision, mais aussi avec les multiples émotions qui l'ont affecté au fil de la situation. Or, de même que le fait d'orienter l'attention vers une douleur corporelle a pour effet de l'amplifier, de même le fait de diriger l'attention d'un interlocuteur vers son intériorité peut avoir pour effet, en cas d'émotion négative, 
de la faire resurgir de manière encore plus vive, avec un risque d'ancrage négatif si par maladresse l'intervieweur maintient le sujet dans l'approfondissement de ce moment difficile. Il faut déjà bien maîtriser la technique des relances en explicitation pour avoir la disponibilité d'esprit permettant de repérer la coloration émotionnelle, positive ou négative, d'une description de pratique, et savoir à la fois identifier une émotion difficile, l'accueillir pleinement, puis relancer sur le fil chronologique de l'action. Une manière simple de le faire est alors de formuler par exemple : "Je comprends, là il y a une partie de toi qui est stressée, et après qu'est-ce que tu fais?".

Donc le premier module est une formation de base à l'explicitation de situations professionnelles positives, avec le début de la consigne conçue par Pierre Vermersch (2015) : "Je te propose, si tu en es d'accord, de prendre le temps de laisser revenir...", suivi d'une orientation quant au contenu : "un moment de pratique professionnelle où ça s'est plutôt bien passé». Le choix de cette formulation atténuée vient du constat qu'en analyse de pratique, non seulement ce sont les situations difficiles qui viennent en premier à la conscience, mais encore qu'il faut souvent un accompagnement très patient pour que les praticiens acceptent l'idée de pouvoir décrire de manière approfondie ce qu'ils savent faire... et qui donc ne leur pose pas question. Les démarches de bilan de compétence (Chauvet, 1997) et de valorisation des acquis de l'expérience (Lainé, 2005) ont montré à quel point ce travail relève d'un accompagnement expert.

Après la formation des étudiants à l'entretien d'explicitation, visant la description de l'activité en situation professionnelle, l'une des directions de travail est précisément de faire suivre le temps descriptif du réfléchissement par un temps réflexif second à partir de la question "Qu'est-ce que tu as su faire?", ce qui permet une verbalisation des savoir-faire incorporés dans la pratique et, le cas échéant, une mise en relation avec la formulation des compétences énoncées dans un référentiel. Afin de bien faire comprendre la distinction entre le temps d'explicitation et le processus d'abstraction qui permet de mettre en évidence les savoirs tacites, je propose d'abord une approche collective en atelier de professionnalisation (Faingold, 2014) puis des mises en œuvre en entretien duel.

Autre chose est de passer, sur la base de cette première description d'un moment de pratique, à un autre type d'accompagnement, l'entretien de décryptage (Faingold, 2011) qui va cette fois chercher, à partir de la position d'évocation et du contact avec le re-vécu de la situation choisie, quels sont les enjeux et les valeurs qui sous-tendent l'activité subjective. Nous nous situons alors à un autre niveau expérientiel, qui est celui de l'identité, et le décryptage est une aide à l'émergence du sens dont l'émotion est le signe. Mais cet accompagnement de la recherche du sens prend deux formes distinctes en fonction de la tonalité émotionnelle de la situation travaillée.

Lorsque le point de départ est une expérience ressource, l'exploration du vécu donne lieu tout d'abord à un recentrage sur un instant identifié par la question : "Dans tout ce que tu viens d'évoquer, quel est l'instant le plus important?". Cette 


\begin{tabular}{|l|l|}
\hline $\begin{array}{l}\text { Première année - Situations ressources } \\
\text { Un moment de pratique professionnelle où ça s'est }\end{array}$ & $\begin{array}{l}\text { Seconde année - Situations difficiles } \\
\text { Une situation professionnelle qui te pose problème }\end{array}$ \\
\hline $\begin{array}{l}\text { FORMATION MÉTHODOLOGIQUE } \\
\text { Apprentissage de la technique d'aide à l'explicitation. } \\
\text { Fondement théorique : P. Vermersch }\end{array}$ & $\begin{array}{l}\text { FORMATION MÉTHODOLOGIQUE } \\
\text { Apprentissage du dispositif de cartographie } \\
\text { émotionnelle } \\
\text { Fondement théorique : R.C. Schwartz }\end{array}$ \\
\hline $\begin{array}{l}\text { DESCRIPTION } \\
\text { Explicitation pas-à-pas du vécu subjectif de } \\
\text { l'activité, mise en évidence de l'enchaînement des } \\
\text { prises d'information et des prises de décision dans } \\
\text { un contexte singulier }\end{array}$ & $\begin{array}{l}\text { DESCRIPTION } \\
\text { Distinction et écriture sur des feuilles distinctes } \\
\text { des éléments de contexte, des déclencheurs, des } \\
\text { émotions impliquées, des croyances sur soi, des } \\
\text { ressources possibles dans une situation singulière }\end{array}$ \\
\hline $\begin{array}{l}\text { RÉFLEXION } \\
\text { Ateliers de professionnalisation } \\
\text { Processus d'abstraction et de mise en mots des } \\
\text { savoir-faire : Qu'est-ce que tu as su faire? } \\
\text { Mise au jour des compétences incorporées }\end{array}$ & $\begin{array}{l}\text { RÉFLEXION } \\
\text { Exploration des parties de soi activées par la } \\
\text { situation, distinction des aspects professionnels et } \\
\text { des éléments de problématique personnelle, mise } \\
\text { en évidence des co-identités }\end{array}$ \\
\hline $\begin{array}{l}\text { ÉMERGENCE } \\
\text { Décryptage du sens: } \\
\text { Les valeurs impliquées } \\
\text { Quel est l'instant le plus important? } \\
\text { Et dans cet instant, qu'est-ce qui est important pour toi? } \\
\text { Le niveau identitaire : Et quand tu fais cela et que } \\
\text { ce qui est important pour toi, c'est..., qui es-tu? }\end{array}$ & $\begin{array}{l}\text { Eù sont les compétences? Les valeurs sous-jacentes? } \\
\text { Quels enjeux identitaires dans cette co-présence de } \\
\text { parties de soi vulnérables et de parties ressource? }\end{array}$ \\
\hline
\end{tabular}

Tableau 1 : Formalisation du dispositif de formation à l'explicitation et au décryptage des pratiques professionnelles

orientation de l'attention mène à l'identification de moments "graines de sens" qui sont le symbole incarné (et souvent exprimé gestuellement) des enjeux et des valeurs qui sous-tendent la pratique. Pour en faire émerger le sens, on procède alors à un arrêt sur image, puis à un patient travail d'aide à la prise de conscience et à la mise en mots de ce qui anime ce moment de vécu, avec un déploiement de l'émotion positive contenue dans ce moment. Il convient de noter que la direction attentionnelle induite par la demande de "l'instant le plus important" suivi de la question "Et dans ce moment, quand tu es là et que tu fais ça, qu'est-ce qui est important pour toi?", conduit le plus souvent à la mise au jour de valeurs essentielles relevant du cœur de métier (Galvani, 2004). En référence aux travaux de Robert Dilts (2006), il est alors possible de poser la question "Et quand ce qui est important pour toi, c'est... qui es-tu à ce moment-là?" qui mène parfois à l'énoncé même de l'identité professionnelle qui s’incarne là de manière singulière.

Quand le point de départ est une situation-problème porteuse d'émotions négatives, le décryptage consiste à établir une cartographie des enjeux identitaires personnels et professionnels co-présents dans le moment exploré. À partir de la description du contexte, l'accompagné est invité à noter comme sur une carte mentale les émotions souvent contradictoires qui le traversent, puis par démarche 
de reprises et de réorganisations successives, à faire émerger les différentes instances identitaires impliquées, les ressources et les fragilités, le personnel et le professionnel. Cette démarche est la transposition en analyse de pratiques de l'approche des parties de soi développée par Richard C. Schwartz (2009) dans le champ de la psychothérapie (Faingold, 2013).

Dans les deux cas, il y a un travail de décryptage du sens qui ne cherche plus à suivre le déroulement chronologique de l'activité, mais à travailler en synchronie pour mettre au jour soit des conflits internes cause de dissonance émotionnelle, soit au contraire un alignement de l'identité et des valeurs, du personnel et du professionnel dans les moments-ressource. Un travail d'aide au changement peut alors s'appuyer sur ces deux modalités d'exploration du sens.

Ce dispositif de formation à l'accompagnement professionnel est donc construit selon deux axes : d'une part une prise en compte de la valence émotionnelle des situations travaillées, et d'autre part une progression qui passe de la description de l'action au décryptage du sens. On peut ainsi formaliser la structure et la progression du dispositif de formation selon un tableau à double entrée (Tableau 1).

Verticalement, ce tableau décline en deux colonnes la progression des mises en situation au fil des deux années du Master. Horizontalement on peut lire en parallèle :

- apprentissage méthodologique et fondement théorique;

- application entre étudiants pour obtenir une description du vécu;

- mise en évidence des compétences sous-jacentes et des constellations identitaires co-présentes;

- accès aux valeurs et au sens qui nous anime, prise de conscience et compréhension des survivances du passé.

Voyons à présent en quoi la forme des exercices proposés favorise à la fois l'apprentissage d'une posture spécifique d'entretien, et la formation à une posture réflexive, conformément aux trois buts de l'entretien d'explicitation et de décryptage : recueil d'information pour l'accompagnateur, apprentissage du geste introspectif et prise de conscience des éléments implicites du vécu pour l'accompagné.

\section{Quelle structure expérientielle des mises en situation pour un développement parallèle des compétences d'accompagnement et des compétences introspectives?}

\subsection{Une formation de base à l'entretien d'explicitation des pratiques}

L'accompagnement en explicitation des pratiques professionnelles est une aide à la prise de conscience par le sujet lui-même de son mode de fonctionnement dans un moment de pratique professionnelle où il est impliqué. Ce qui est attendu est une verbalisation en première personne, en "je», à partir de la position d'évocation, définie 
par Pierre Vermersch (1994) comme cette position de parole spécifique où le sujet est davantage tourné vers son monde intérieur que vers l'interlocuteur, davantage présent à la situation passée qu'à la situation actuelle de communication. Ce point est essentiel, puisqu'il pose la nécessité de prendre le temps du processus de réfléchissement qui remet en contact avec le vécu passé dans un "quasi-revivre", avant toute démarche de réflexion qui consiste à revenir sur des éléments déjà conscientisés.

Les étudiants travaillent systématiquement par groupes de trois et peuvent alternativement prendre trois rôles différents :

- l'intervieweur est en apprentissage de l'entretien d'explicitation des pratiques;

- l'interviewé fait l'expérience de la position d'évocation, de la richesse des remémorations possibles, et il apprend les actes de l'exploration introspective, comme la visée à vide, le laisser-venir, l'intention éveillante, la sollicitation de la mémoire passive (Vermersch, 2006, 2012);

- l'observateur a le privilège de développer son aptitude à prendre de l'information, ce qui lui permettra ultérieurement, en situation d'entretien, à la fois d'intégrer un mode de questionnement et de prendre en compte beaucoup plus finement les indices paraverbaux et non verbaux manifestés par son interlocuteur.

\subsubsection{Du côté de l'intervieweur}

Les premières mises en situation ont pour but de mettre en place la distinction entre un récit spontané de pratique adressé à autrui, et la description pas-à-pas, en position introspective, de la complexité de ce qui se joue subjectivement en cours d'activité. Pour l'intervieweur, il s'agit de repérer les verbalisations qui relèvent du contexte (environnement, lieu, circonstances, mais aussi et surtout action d'autrui), et celles qui relèvent de commentaires au sujet de la situation, pour, dans un cas comme dans l'autre, réorienter le sujet vers le contact avec son vécu passé et obtenir une description du détail de son action. En entretien, le but sera de systématiquement relancer vers l'univers subjectif. Les relances seront du type : "quand tu es là et qu'il se passe cela, et qu'il (autrui) fait cela... toi, qu'est-ce que tu fais?" (ré-orientation du contexte ou de l'action d'autrui vers l'action du sujet). Mais aussi : "Tu penses donc que c'était une bonne idée (reprise en écho d'un commentaire). Et maintenant, si tu en es d'accord, je te propose de revenir au moment où..." (ré-orientation vers l'activité). Parallèlement se met en place la distinction entre la position de parole habituelle et la position d'évocation, qui peut être obtenue par sollicitation de la mémoire corporelle et sensorielle de la situation, et par une incitation constante au ralentissement, pour obtenir le déploiement de toutes les facettes du vécu.

Mais en termes de posture d'accompagnement, les déplacements les plus importants s'opèrent au fil de l'appropriation de la technique même de l'explicitation, qui nécessite un effacement de toute tentation interprétative pour mettre en place ce que j'ai appelé une "écoute vide", condition d'un accueil inconditionnel de la 
singularité du vécu de l'autre. Cette écoute spécifique instaure la confiance, autorise l'authenticité, et crée ainsi pour l'autre l'espace possible d'une "parole pleine". C'est en apprenant à poser des questions non inductives, à reprendre exactement les mots de l'autre avant d'enchaîner par une question d'approfondissement, que les étudiants comprennent expérientiellement ce qu'est un accompagnement : partir toujours de là où en est l'autre pour le faire avancer vers lui-même.

\subsubsection{Du côté de l'interviewé}

Ce qui se joue pour les personnes qui expérimentent la position d'évocation, c'est qu'ils ont à lâcher prise par rapport à leurs habitudes de rationalisation et de contrôle, à accepter de se faire accompagner, ce qui n'est pas si simple et nécessite de la part des intervieweurs une vérification constante du contrat de communication. Seuls une relation de confiance et un sentiment de sécurité qui se renforcent à mesure que le groupe apprend à se connaître permettent une parole subjective authentique, enracinée dans le vécu. Les interviewés vivent aussi en direct les effets perlocutoires (Vermersch, 2012), à savoir ce que nous font les mots de l'autre, et spécifiquement les incidences émotionnelles souvent violentes des questions inductives, intrusives ou porteuses de jugements implicites, comme le banal et redoutable "Pourquoi?". Mais l'effet principal de l'expérience de l'évocation et de l'exploration de l'univers subjectif est que l'on y prend goût et que très rapidement les interviewés vont demander à celui qui mène l'entretien de mieux questionner, de mieux relancer, de mieux induire le ralentissement, d'approfondir le contact avec le vécu exploré. Comme le remarque Pierre Vermersch dans un texte de son blog (Vermersh, 2014) : "Pour certains, cette formation personnelle est un choc, une découverte, une rencontre recherchée depuis longtemps, un cadeau de découvrir que c'est possible de décrire son monde intérieur, d'y rester, d'y retourner".

\subsubsection{Du côté de l'observateur}

L'observateur peut avoir une consigne lui demandant de porter son attention sur les indicateurs de la position d'évocation chez l'interviewé (décrochage du regard, coloration émotionnelle, gestuelle, ralentissement du rythme de la voix), et de repérer les sorties d'évocation. Dans une autre mise en situation, il peut avoir pour consigne de porter son attention sur l'accompagnement de l'intervieweur, et dans ce cas, sachant qu'il est libéré du souci de chercher la bonne relance et qu'il voit clairement ce qu'il conviendrait de faire, l'un des points importants sera d'apprendre à faire à son collègue un feedback à la fois précis et bienveillant, et de travailler dans ce cadre l'attention aux effets perlocutoires de ses remarques.

Nous venons de développer ce qui nous a semblé être le meilleur point de départ d'une formation à l'accompagnement professionnel en relation duelle : l'apprentissage d'une posture nouvelle d'écoute et d'aide à la prise de conscience à travers la formation de base à l'entretien d'explicitation. Ce dispositif de mise en situation expérientielle par groupe de trois (parfois quatre) étudiants est le même dans les exercices visant la mise au jour des compétences et/ou le décryptage du sens. Il est par ailleurs à chaque fois 
possible, après un exercice, d'enchaîner par un entretien sur l'entretien, qu'il s'agisse d'aider un intervieweur à revenir sur la manière dont il a conduit l'entretien, de proposer un travail psycho-phénoménologique de description des actes de conscience opérés par celui qui évoque, ou encore de permettre à un observateur de décrire son vécu au fil des interactions dont il a été le témoin. Précisons que toutes les mises en situation sont enregistrées, et le cas échéant transcrites quand l'étudiant choisit de les analyser pour un travail écrit en vue d'une évaluation. Nous souhaitons à présent illustrer ce qui se joue pour les étudiants en termes de formation et de transformation de soi par des exemples issus de leurs travaux écrits.

\section{2. Écrits réflexifs d'étudiants portant sur des temps d'accompagnement} professionnel

Les deux exemples que nous allons développer sont extraits de travaux rendus par des étudiants du Master à des fins d'évaluation de leurs compétences d'accompagnement en utilisant l'approche de l'entretien d'explicitation et de décryptage. Le premier écrit est celui d'un formateur qui restitue l'apport d'un entretien mené auprès d'une collègue enseignante, et opère ensuite un retour réflexif sur sa posture d'accompagnement au cours de cet entretien (lors d'une séance de formation à l'université en première année du master). Le second exemple (issu d'un écrit de fin de seconde année) rend compte d'un accompagnement en plusieurs temps, permettant la mise au jour des enjeux identitaires impliqués dans le parcours de formation d'une jeune infirmière, et permet de comprendre comment peuvent se construire des compétences réflexives chez les personnes accompagnées.

\subsubsection{Olivier, intervieweur et interviewé lors d'une mise en situation à l'Université}

Olivier est professeur-formateur académique, il mène un entretien avec Sylvie, enseignante en économie gestion. Elle a deux classes en binôme avec deux collègues différents, et il s'agit de constituer pour chaque élève des dossiers en vue d'une évaluation assez complexe, portant sur des fiches dans trois domaines différents. Un premier entretien d'explicitation permet à Sylvie de décrire la manière dont elle a fonctionné avec des codes couleur pour organiser des dossiers et des sous-dossiers. Nous sommes ici à un niveau d'aide à la description de l'activité en fonction d'un premier but opérationnel conscient.

Olivier l'accompagne ensuite pour que soit mis en évidence l'intérêt professionnel de cette action et la finalité sous-jacente, ce qui permet à Sylvie d'exprimer : "Ce qui s'avère pratique c'est d'avoir fait un dossier par élève où lui-même peut répertorier son travail, et pour les professeurs un dossier avec un tableau récapitulatif par classe, du coup tout le monde est autonome". Ce qui est rendu possible, à travers l'aspect "pratique" de l'outil qu'elle a créé, c'est donc l'autonomie de chacun et spécifiquement des élèves en tant que visée pédagogique essentielle. 
Vient ensuite un second temps de l'entretien, visant à mieux cibler le moment porteur d'enjeux identitaires pour aller vers un décryptage du sens.

À la question "Est-ce qu'il y a un moment, un instant plus important?", Sylvie répond: "Oui, en fait c'est le moment où je me suis posée, où j'ai pris le temps vraiment de réfléchir comment j'allais organiser le dossier. " Notons que dans un premier temps, on ne voit pas a priori d'enjeu identitaire dans cette citation. Par expérience, Il faut faire confiance dans le fait que le choix du moment par le sujet luimême est un élément décisif : l'expérience prouve que l'instant qui émerge en réponse à cette question visant "ce qui est important" est porteur d'un sens encore implicite dont le sujet détient seul la clé, mais qu'il découvrira lui-même au fil de l'entretien dans une démarche maïeutique (Faingold, 2011). Olivier mène alors un nouveau temps d'explicitation sur ce moment précis de la conception des dossiers, et Sylvie retrouve ce qui a été déterminant : elle a devant elle à gauche le référentiel avec les tâches énumérées dans un tableau, et à droite les pochettes, elle prend le temps de regarder, et elle voit alors dans l'espace comment elle va pouvoir transposer de l'un à l'autre. Il s'agit en fait d'un véritable moment de créativité.

La suite de l'entretien correspond au temps de décryptage, avec maintien en prise sur l'évocation du moment, et accompagnement corporel et gestuel par Olivier. Les passages entre crochets décrivent les aspects non verbaux de l'interaction.

Olivier : Et ça, c'est le moment le plus important pour toi?

Sylvie : Oui... Je me suis dit : "c'est ça que je veux et c'est comme ça que ça va marcher!"

0 . : D'accord et tu l'as ressenti corporellement?

S. : Oui... je me suis dit : "ça y est..."

[Sylvie a recontacté le souvenir et la sensation corporelle. Elle indique son ventre avec ses mains] je savais que c'était ça, ce à quoi je voulais aboutir... Mon outil à moi, en fait.

0. : Et quand t'as senti là

[Olivier reprend lui aussi le geste avec ses mains montrant son ventre] t'as senti que c'était ça?

S. : Oui!

[Sylvie a un très grand sourire et le regard illuminé]

0. : Qu'est-ce qu'il y avait ici?

S. : C'est une boule en fait... c'est quelque chose qui est là...

0 . : d'accord... et là, tu sais que c'est ça?

[Olivier reprend son geste et pendant cette reprise du geste, Sylvie est radieuse]

S. : Et là je sais que j’ai bien bossé... Merci Olivier...

Par ces mots très simples "j'ai bien bossé" c'est la satisfaction profonde d'avoir fait son métier qu'exprime Sylvie, d'avoir créé quelque chose qui lui est propre, et d'avoir œuvré pour les autres. 
Après une pause, une autre étudiante, Cécile, va mener un entretien d'explicitation et de décryptage sur le vécu d'Olivier au cours de cet accompagnement. Les premières relances de Cécile ne sont pas transcrites. Les commentaires d'Olivier formulés $a$ posteriori dans son travail écrit sont entre crochets.

Olivier : Alors, le moment sur lequel j'aimerais revenir, c'est le moment où j'arrive à, à remettre Sylvie en contact avec l'émotion positive très forte qu'elle a ressentie au creux d'elle-même... J'essaie de la ralentir, elle repart en évocation, je commence à sentir que c'est vraiment ce moment-là qui est le plus riche pour elle, j'ai l'impression de la comprendre j'ai l'impression d'être avec elle... J'ai l'impression de ne plus m'intéresser à ce que je ressens moi, et je suis complètement à l'écoute de ce qu'elle peut me dire ou de ce qu'elle peut me montrer et je... moi je ne suis plus là... Je ne suis qu'avec elle, voilà, et quand je suis avec elle du coup j'ai des questions qui viennent toutes seules... Je suis sûr de moi... Je n'hésite plus, tout se met en place... Et du coup, voilà, je ne suis qu'avec elle.

Il se joue ici quelque chose qui me rassure et me donne confiance... Le sentiment que je n'ai plus besoin de rien, une forme de fusion archaïque... Comme entre un nouveau-né et sa mère... Quand elle me dit : "C'est comme un Soleil"... c'est quelque chose qui diffuse comme ça partout j'ai l'impression de le ressentir moi... il y a eu un moment on faisait les gestes en même temps et c'était magique, c'était comme si on était... sur la même longueur d'onde... c'était un bon moment...

[Je prends conscience que c'est ce qui m'attire dans mon travail. C'est ce qui fait que j'ai choisi des métiers de la relation. En même temps, je suis très proche aussi des émotions négatives associées, à savoir du vide en moi que je cherche à combler par mes activités professionnelles mais également personnelles. Cela me questionne... en même temps que cela vient d'apparaitre par l'évocation...]

Cécile reprend pour approfondir encore et demande...

Cécile : Dans ce moment qu'on vient d'explorer, quel est le moment le plus important?

Olivier : Le moment où il y a un peu une fusion entre Sylvie et moi.

C. : Et quand tu es là qu'est-ce qui est important pour toi au moment de la fusion?

0. : Qu'est-ce qui est important? II n'y a rien d'important, justement... c'est pas «important ou pas important", c'est juste un instant... je sais pas comment dire, c'est un peu un instant... d'éternité quoi.

C. : un instant d'éternité...

0. : Oui, quelque chose comme ça, un petit instant... c'est difficile d'exprimer avec des mots...

[Je sens l'émotion très profondément en moi, cela me renvoie à quelque chose que j’ai ressenti il y a très longtemps, très loin dans mon enfance]

Oui, ce qui se passe c'est que je ne suis plus en contact avec mon corps, je ne suis plus en contact avec ma pensée je suis vraiment en train de vivre l'émotion avec Sylvie... et du coup c'est agréable...

[En fait, je suis en contact non plus avec l'émotion de Sylvie mais avec l'émotion pure de fusion. Je prends conscience que c'est quelque chose que je recherche de façon récurrente et que j'atteins ici. Mais dans le même temps, cela me renvoie un peu à quelque chose qui s'est passé il y a très longtemps... Cette émotion de fusion a beaucoup de sens pour moi. Inconsciemment, elle organise ma vie depuis des années et à ce moment le fait de ressentir cette émotion est positif mais en même temps elle renvoie à des choses qui font que j'ai un vide affectif à combler par les relations professionnelles. Je viens d'en 
prendre conscience. Des choses qui font que je préfère vivre à travers l'autre, que je cherche à être l'autre et non pas à être moi. Des choses qui font que je m'investis autant professionnellement. Cet entretien d'explicitation m'ouvre les yeux sur un fonctionnement de moi dont je n'avais pas conscience. En conclusion, ces deux entretiens constituent une sorte d'électrochoc. Par ce processus de recherche inconsciente de fusion, devenir l'autre en tant que formateur, c'est perdre le rôle d'accompagnateur, c'est perdre sa distance psychique qui permet de guider l'autre. Il est important que je travaille sur ce point. Cela rejoint ce qu'écrivait Carl Rogers (1961) : "Suis-je suffisamment moi-même, intégré et unifié, pour être alors perçu comme sécurisant par autrui?".]

L'exemple ci-dessus est issu de la transcription d'une séance de formation à I'Université. II montre en quoi l'entretien d'explicitation contribue à la fois au développement des compétences d'accompagnement et à la prise de conscience de soi. L'intérêt de la formation est évidemment le réinvestissement que nos étudiants en font sur leur terrain professionnel pendant la seconde année du master, et dont ils rendent compte dans leurs écrits de seconde année. Dans l'exemple qui suit, il s'agit de l'accompagnement professionnel d'une étudiante en Institut de formation aux soins infirmiers (IFSI) par une formatrice, cadre de santé, inscrite en Master.

\subsection{2. Écrit réflexif sur un accompagnement professionnel : Anne et Laïla}

Anne mène avec Laïla, étudiante infirmière, un entretien d'explicitation et de décryptage, qui va permettre la description d'une situation de soin, puis la mise au jour des parties de soi impliquées. Laïla réalise un soin infirmier assez délicat auprès d'une patiente âgée porteuse d'une trachéotomie et qui a une gêne respiratoire. Tout en décrivant le détail des gestes qu'elle réalise, elle identifie une partie d'elle qui a peur, et une partie d'elle qui doit montrer que tout va bien.

Laïla : En fait, en la rassurant elle (la patiente), je me rassure moi. Et quand je vois qu'elle est apaisée, ça m'apaise.

Anne : Et quand elle s'apaise, vous êtes qui à ce moment-là?

L. : Une infirmière. Oui, une infirmière calme... Parce que je sens qu'il y a une confiance... C'est surtout son regard... Un regard doux qui a l'air de dire : "Allez-y, je vous fais confiance"... À ce moment-là, je me dis : "il ne faut pas que je me loupe..."

A. : Donc il y a une partie qui dit "il ne faut pas que je me loupe", et l'autre partie, qu'est-ce qu'elle dit?

L. : Elle me dit j'ai confiance en moi...

A. : Quand il y a cette relation de confiance, vous vous sentez comment?

L. : Je me sens infirmière. En fait, "il ne faut pas que je me loupe", c'est plus mon côté étudiante. Et après, tout de suite je reprends le contrôle : ce geste, je le connais.

Anne [commentaire dans son écrit réflexif] : "Dans le cadre de mon mémoire et donc de l'accompagnement de cette étudiante, j'ai pris le parti de travailler les après-coups de deux façons différentes. D'une part en lui demandant un écrit sur le prolongement de sa réflexion après l'entretien, et d'autre part en lui donnant les verbatim à lire puis en formalisant un temps de rencontre pour revenir sur sa perception des éléments livrés lors de l'entretien... Un des effets directs de l'entretien 
sur son lieu de stage a été l'adoption d'une posture plus réflexive dans les heures qui ont suivi. Nous avions abordé ensemble la place qu'elle occupait en tant qu'étudiante, sa difficulté à trouver sa place de professionnelle auprès des médecins, et surtout sa posture vis-à-vis de certaines personnes, face auxquelles elle se sentait parfois intimidée au point de "parler comme une petite fille" et sur la prise de distance dans le vécu de certaines relations à l'Autre."

À côté des co-identités (Faingold, 2001, Snoeckx, 2011, Vermersch 2011) "étudiante" et "infirmière", on voit intervenir ici la co-identité "petite fille" intimidée par la figure d'autorité, ce qui est souvent le cas chez les débutants qui n'ont pas encore investi leur place professionnelle.

Quand Anne la revoit, Laïla décrit un moment où un patient parle à l'infirmière et ne la regarde pas parce qu'elle est étudiante.

Laïla : "J'étais à côté. Et là, j'étais en train d'analyser ce que vous étiez en train de me dire. À quel moment j'ai ma place, est-ce que j'ai ma place ou est-ce que je n'ai pas ma place? Et en fait, le patient était en train de parler à l'infirmière, j'écoutais tout ce qu'ils étaient en train de se dire mais là je me disais bon, ben voilà, là, je n'ai pas ma place et on voit bien... la barrière en fait. Et là, j'ai analysé, j'ai réfléchi [...] J'ai compris pourquoi : c'était l'infirmière et pas moi. Peut-être que dans un autre moment... je me serais sentie à l'écart. Mais là, non...".

Anne commente ensuite au plan théorique ce nouveau pas dans l'avancée de Laïla vers une posture de praticien réflexif (Schön, 1994).

Anne : "L'adoption de cette posture réflexive à ce moment précis a semble-t-il été favorisée par tout le travail de réflexion effectué quelques heures plus tôt, comme si cette séance en face à face avait créé les conditions d'une prise de distance suffisante pour adopter un regard distancié... Peut-on alors parler de mise en place d'un schème réflexif à ce moment-là? Et si oui, peut-on supposer qu'il restera suffisamment stable pour contribuer à l'adoption d'une posture réflexive à plus long terme? Dans son retour écrit, Laïla évoque une situation analogue dans laquelle elle s'est retrouvée le lendemain de ma visite et de l'entretien d'explicitation : "j'ai alors, malgré moi, analysé mon soin : j'ai recherché mes émotions, à quel moment je ressentais de la peur, à quel moment j'étais satisfaite. J'ai cherché le regard de la patiente pour tenter de savoir son ressenti". La précision de ce "malgré moi" me semble intéressante parce qu'elle laisse entrevoir qu'il n'y a pas eu d'intention préalable à l'analyse du soin. Le regard introspectif sur son agir se serait imposé à elle. Je fais l'hypothèse que l'analyse antérieure du même soin sous forme d'explicitation a permis ce regard distancié, cette forme de rapport réflexif à la situation... Réaliser un geste, reconvoquer son vécu à distance, prendre conscience des postures tant gestuelles que psychiques contribue, à mon sens, à l'installation de schèmes réflexifs et de ce fait, à la formation d'un habitus réflexif."

Selon moi, le remarquable travail d'accompagnement professionnel effectué par Anne en proposant successivement à Laïla différentes modalités de retours réflexifs, condense la plupart des compétences travaillées dans le dispositif, et l'analyse qu'elle en fait illustre bien la transférabilité de situations de formation vécues à l'Université au terrain professionnel. 


\section{En guise de conclusion}

Pour conclure cette description d'un dispositif de formation à l'accompagnement professionnel, je voudrais remercier l'ensemble de mes étudiants du Master, j'ai relu une grande partie de leurs travaux avant de rédiger cet article, et je n'en ai finalement cité que deux². Mais à tous je voudrais dire que c'est grâce à eux, à leurs interrogations, à leurs remarques, que ce dispositif s'est affiné au fil du temps, de manière implicite, sans que je sache moi-même toujours très clairement comment s'articulaient les différentes unités d'enseignement que m'imposait le découpage universitaire, entretien d'explicitation, entretien de décryptage, ateliers de professionnalisation, aide au changement, exploration des parties de soi, approfondissements optionnels, séminaire de recherche sur les enjeux identitaires en formation... II a fallu cet article pour qu'au terme de mes relectures se dessine et se clarifie le schéma qui rend compte de la logique sous-jacente de la progression suivie. Une autre découverte en revisitant leurs travaux, a été la formulation récurrente chez beaucoup d'étudiants de ce qui a été pour eux un facteur essentiel de changement : l'apprentissage du lâcher-prise, qui apparaît ainsi comme l'une des clés décisives de la transformation de soi et de l'ouverture à l'autre. Dans la tentative de rendre explicite et transmissible ce qui est de l'ordre d'un vécu partagé il est donc temps pour moi aussi de lâcher prise et de laisser la place aux questions des lecteurs, qui ouvriront sans doute de nouvelles pistes de recherche en formation.

Nadine Faingold nadine.faingold@gmail.com

\section{Bibliographie}

Chauvet A. (1997). Bilans de compétences et aide à l'explicitation. In P. Vermersch et M. Maurel (dir.), Pratiques de l'entretien d'explicitation. Paris: ESF.

Dilts, R. (2006). Changer les systèmes de croyances avec la PNL. Paris : Interéditions.

Faingold, N. (2001). Identifier les co-identités. Expliciter, 41, 10-14. En ligne, consulté en septembre $2016:<w w w . g r e x 2 . c o m>$.

Faingold, N. (2011). L'entretien de décryptage : le moment et le geste comme voies d'accès au sens. Expliciter, 92, 24-47.

Faingold, N. (2012). La formation à l'entretien d'explicitation comme recherche-action sur soi, Expliciter,89, 15-25.

Faingold, N. (2013). Accompagner l'émotion : explicitation, décryptage du sens et parties de soi, Expliciter, 100, 29-38.

Faingold, N. (2014). Réduction et résonances en recherche et en formation : des compétences en acte à l'identité professionnelle. In A. Mouchet (dir.), L'entretien d'explicitation. Usages diversifiés en recherche et en formation (p.39-60). Paris : L'Harmattan.

2 Un merci tout particulier à Olivier Mathieu et Anne Millot, qui m’ont autorisée à publier de larges extraits de leurs travaux. 
Faingold, N. (2015). De l'explicitation des pratiques professionnelles au décryptage du sens. En ligne, consulté en octobre 2015 : <http://www.grex2.com/assets/files/ Dossiers/Dossier\%20Nadine\%206\%20septembre.pdf>.

Galvani, P. (2004). L'exploration des moments intenses et du sens personnel des pratiques professionnelles, Interactions, 8 (2).

Galvani, P. (2014). L'accompagnement maïeutique de la recherche-formation en première personne, Éducation permanente, 201, 98-112.

Lainé, A. (2005). VAE, quand l'expérience se fait savoir. L'accompagnement en validation des acquis. Paris : Éditions ERES.

Rogers, C. (1961). Le développement de la personne. Paris : Dunod.

Schön, D. (1994). Le praticien réflexif. À la recherche du savoir caché dans l'agir professionnel. Montréal, Canada : Logiques.

Schwartz, R.C. (2009). Système familial intérieur. Blessures et guérison. Issy-lesMoulineaux : Elsevier Masson.

Snoeckx, M. (2011). Une contribution à la réflexion à propos des co-identités. Expliciter, 90, 1-12.

Vermersch, P. (1994). L'entretien d'explicitation. Paris : ESF.

Vermersch, P. (2006). Rétention, passivité, visée à vide, intention éveillante. Expliciter, $65,14-28$.

Vermersch, P. (2011). Note préparatoire sur le thème des co-identités. Expliciter, 90, 13-18. Vermersch, P. (2012). Explicitation et phénoménologie. Paris : PUF.

Vermersch, P. (2014). Double apprentissage. En ligne, consulté en septembre 2016 : $<$ http://www.entretienavecpierre.fr/2014/05/double-apprentissage/>.

Vermersch, P. (2015). L'entretien d'explicitation et la mémoire (2). Expliciter, 107, 44-45.

\section{Abstract}

\section{A university scheme focused on reflective work exploring subjective experience: education and self-transformation}

ABSTRACT: Cergy-Pontoise University's Master's in "Training trainers in vocational support" is designed for professionals in occupations which require people skills, and favours a first person approach which is experiential and reflective. In this academic context, we propose a scheme which focuses on the elicitation of subjective experience and the description of issues and values involved in professional practices. Students are encouraged to develop vocational support including elicitation and description interviews, and then provide reflective feedback on their stance and their own subjective experience during this support.

KEYWORDS: trainer, professional experience, interpersonal relations, adult education, méthodology 
